\title{
Sequential Nuclear Accumulation of the Clock Proteins Period and Timeless in the Pacemaker Neurons of Drosophila melanogaster
}

\author{
Orie T. Shafer, ${ }^{1}$ Michael Rosbash, ${ }^{2}$ and James W. Truman ${ }^{1}$ \\ ${ }^{1}$ Department of Zoology, University of Washington, Seattle, Washington 98195, and ${ }^{2 H o w a r d}$ Hughes Medical Institute, \\ National Science Foundation Center for Biological Timing, and Department of Biology, Brandeis University, Waltham, \\ Massachusetts 02254
}

\begin{abstract}
Antisera against the circadian clock proteins Period (PER) and Timeless (TIM) were used to construct a detailed time course of PER and TIM expression and subcellular localization in a subset of the ventrolateral neurons (vLNs) in the Drosophila accessory medulla (AMe). These neurons, which express pigmentdispersing factor, play a central role in the control of behavioral rhythms. The data revealed several unexpected features of the circadian clock in Drosophila. First, TIM but not PER was restricted to the cytoplasm of vLNs throughout most of the early night. Second, the timing of TIM and PER nuclear accumulation was substantially different. Third, the two subsets of
\end{abstract}

vLNs, the large and small vLNs, had a similar timing of PER nuclear accumulation but differed by 3-4 hr in the phase of TIM nuclear accumulation. These aspects of PER and TIM expression were not predicted by the current mechanistic model of the circadian clock in Drosophila and are inconsistent with the hypothesis that PER and TIM function as obligate heterodimers. The differing profiles of TIM and PER nuclear accumulation suggest that PER and TIM have distinct functions in the nuclei of vLNs.

Key words: Period; Timeless; pigment-dispersing factor; circadian rhythm; Drosophila; lateral neurons; nuclear accumulation
Transcriptional feedback loops are a common theme underlying circadian clocks from many different organisms (Dunlap, 1999). These loops typically include components that positively or negatively regulate the transcription of other core clock genes. They also include delay mechanisms that make possible the robust, near $24 \mathrm{hr}$ molecular oscillations that characterize circadian rhythms.

The clock genes period (per) and timeless (tim) encode key components of the circadian clock of Drosophila melanogaster (Williams and Sehgal, 2001). The products of these genes are required for locomotor rhythms and are expressed rhythmically in the fly (Konopka and Benzer, 1971; Hardin et al., 1990; Zerr et al., 1990; Sehgal et al., 1994, 1995). Period (PER) and Timeless (TIM) proteins are believed to inhibit their own transcription through their interactions with the positive transcription factors Clock and Cycle (Allada et al., 1998; Darlington et al., 1998; Rutila et al., 1998; Lee et al., 1999). The regulation of PER and TIM nuclear entry is believed to provide an important delay between the synthesis and accumulation of these proteins and their subsequent inhibitory activity in the nucleus (Vosshall et al., 1994; Curtin et al., 1995; Saez and Young, 1996). Recent data suggest that the regulation of PER nuclear entry is also important for the mammalian clock (Lee et al., 2001). In flies, the formation of PER/TIM heterodimers is thought to be a key event regulating

Received Jan. 22, 2002; revised April 29, 2002; accepted May 1, 2002

This work was supported by United States Public Health Service National Research Service Award T32 GM07270 from the National Institute of General Medical Sciences (O.T.S.), by a grant from the National Science Foundation (NSF) Center for Biological Timing (M.R.), and by NSF Grant 1BN0080894 (J.W.T.). We thank K. R. Rao for providing cPDH antisera, Michael Young and Amita Sehgal for kindly providing TIM antisera, and Jeff Hall, Joel Levine, and Darren Williams for helpf ul discussions and comments on this manuscript.

Correspondence should be addressed to Orie Shafer, Department of Zoology, University of Washington. Box 351800, 24 Kincaid Hall, Seattle, WA 98195. E-mail: oshafer@u.washington.edu.

Copyright (C) 2002 Society for Neuroscience $\quad 0270-6474 / 02 / 225946-09 \$ 15.00 / 0$ protein accumulation and nuclear entry (Zeng et al., 1996; Suri et al., 1999). This conclusion is supported by studies in cultured S2 cells in which PER and TIM appear to enter the nucleus as a heterodimeric complex (Saez and Young, 1996).

These observations make clear predictions about the pattern and timing of PER and TIM nuclear accumulation during a circadian cycle in flies. However, most studies addressing the expression and nuclear accumulation of PER and TIM have used biochemical extracts of whole-head homogenates or cell lines that do not produce overt rhythms. These studies have therefore not afforded a complete picture of PER and TIM dynamics within individual clock-containing cells.

A key set of clock-containing neurons are the large and small ventrolateral neurons (vLNs) of the accessory medulla (AMe), which are critical for robust locomotor rhythms (Ewer et al., 1992; Hardin et al., 1992; Frisch et al., 1994; Helfrich-Forster, 1998; Renn et al., 1999). The only study with an hourly time course of PER dynamics in these cells was performed before the availability of anti-TIM reagents or of an independent marker for the vLNs (Curtin et al., 1995). To compare the timing of PER and TIM nuclear localization and to test additional predictions of the present molecular model, we set out to describe the dynamics of PER and TIM expression in these pacemaker neurons. In contrast to expectation, our results indicate that PER and TIM show different profiles of nuclear accumulation in wild-type flies. The large and small vLNs also show differences in the timing of PER, and to an even greater extent TIM nuclear entry, which underscores the notion that the nuclear accumulation of these two proteins is not tightly coupled.

\section{MATERIALS AND METHODS}

Fly stocks and entrainment. Canton S (wild type) and the genetically null mutant strains $\mathrm{per}^{01} ; \mathrm{ry}^{506}$ and $\mathrm{tim}^{01} ; \mathrm{ry}^{506}$ of D. melanogaster were reared at room temperature on cornmeal-molasses medium. Before dissection, flies were entrained for $3-5 \mathrm{~d}$ in a $12 \mathrm{hr}$ light/dark (LD) cycle at $25 \pm 1^{\circ} \mathrm{C}$ 
in light boxes housed in an environmental chamber. Flies were typically 1-2 d old at the start of entrainment and 4-7 d old at the time of dissection.

Dissection. Brains were dissected in 15 min windows centered on each reported Zeitgeber time (ZT), where ZT 00/24 is lights-on and ZT 12 is lights-off in a $12 \mathrm{hr}$ LD cycle. Tissue used for PER and TIM staining at a particular ZT was dissected from the same vial of flies for any given time point. Flies were pinned down in a sylgard dish (Dow Corning, Midland, MI) under PBS by means of a small insect pin through the thorax. The head cuticle was quickly torn using two fine forceps, exposing the underlying brain. Partially dissected heads were removed and placed in a round bottom $2 \mathrm{ml}$ Eppendorf tube filled with room temperature $3.7 \%$ formaldehyde in PBS. Brains for PER and TIM staining at a particular ZT were placed in separate tubes. After all heads of a given time point were in fix, tubes were placed on ice for $5 \mathrm{~min}$ to encourage the heads to sink and gently agitated for $20 \mathrm{~min}$ at room temperature. After this initial fixation, heads were rinsed quickly in PBS and dissected to remove the cuticle and pigmented eye tissue. Brains were then postfixed at room temperature with agitation for $15 \mathrm{~min}$, rinsed in PBS, and placed in PBS plus $0.3 \%$ Triton X-100 (PBS-TX) on ice to await blocking.

Immunocytochemistry. Brains were blocked in $2 \%$ normal donkey serum in PBS-TX for $30-40 \mathrm{~min}$ at room temperature. After block, tissue was rinsed in PBS-TX and placed in $50 \mu \mathrm{l}$ of primary antisera solution for two nights at $4^{\circ} \mathrm{C}$. Rabbit anti-PER (So and Rosbash, 1997) and rat anti-TIM antisera (generous gifts from A. Sehgal, University of Pennsylvania, Philadelphia, PA, and M. Young, Rockefeller University, New York, NY) were diluted at 1:1000 in PBS-TX. Rat anti-pigmentdispersing factor (PDF) (Renn et al., 1999) was used at either 1:500 or 1:1000 dilutions, and rabbit anti-crustacean ortholog pigment-dispersing hormone (cPDH) (generous gift from K. R. Rao, University of West Florida, Pensacola, FL) (Dircksen et al., 1987; Helfrich-Forster, 1995) was used at a 1:20,000 dilution in PBS-TX. Rat anti-PER and rabbit anti-TIM antisera (raised in the laboratory of M. Rosbash) were also used at 1:1000 for the replicate time courses. After exposure to the primary antisera, brains were rinsed five times for 15 min each in PBS-TX on a rotator. Tissue was then placed in secondary antisera for one night at $4^{\circ} \mathrm{C}$. For PDF and PER staining, 1:1000 dilutions of FITCconjugated donkey anti-rat and Texas Red-conjugated donkey anti-rabbit (Jackson ImmunoResearch, West Grove, PA) in PBS-TX were used. For cPDH and TIM staining, 1:1000 dilutions of FITC-conjugated donkey anti-rabbit and Texas Red-conjugated donkey anti-rat in PBS-TX were used. After exposure to the secondary antisera, brains were rinsed five times for 15 min each in PBS-TX, rinsed in PBS, mounted on poly-Llysine-coated coverslips, dehydrated in a graded EtOH series, and cleared in two rinses of xylene. Brains were mounted in DPX mounting medium (Fluka, Milwaukee, WI) between two coverslips.

Confocal microscopy. Optical sections of vLNs were imaged on a Bio-Rad (Richmond, CA) MRC 600 confocal microscope. For each neuron sampled, PDF or cPDH staining was used to identify the appropriate neuron and to select an optical section that included the nucleus. The same optical section was then scanned for PER or TIM immunoreactivity. Merged images were constructed in Adobe Photoshop (Adobe Systems, San Jose, CA). Other than the addition of color and the merging of the FITC and Texas Red images, no manipulations of original confocal images were executed in Photoshop. Sample micrographs were chosen to represent as closely as possible the means of two separate measurements: cytoplasmic and nuclear pixel intensities (see below). For this reason it was sometimes necessary to present an image with a cytoplasmic or nuclear value that lies near but not beyond the maximum or minimum values described by the error bars in the quantifications (see below).

Quantification of staining. Confocal files were imported to NIH Image 1.62 (available by anonymous FTP from zippy.nimh.nih.gov/pub/nihimage). The images were inverted, and the location of the cytoplasm and nucleus of each neuron was determined by the PDF/cPDH image. For each vLN cell body, mean pixel intensity of PER or TIM staining was measured for the cytoplasm, nucleus, and background. Net cytoplasmic and nuclear pixel intensities were calculated by subtracting the mean background pixel intensity from the cytoplasmic and nuclear measurements. Mean pixel intensity was used as a measure of relative staining intensity and therefore the relative amount of protein present in the cytoplasm and nucleus. Minimum and maximum pixel intensities were 0 and 255, respectively. The mean pixel intensity was determined for all

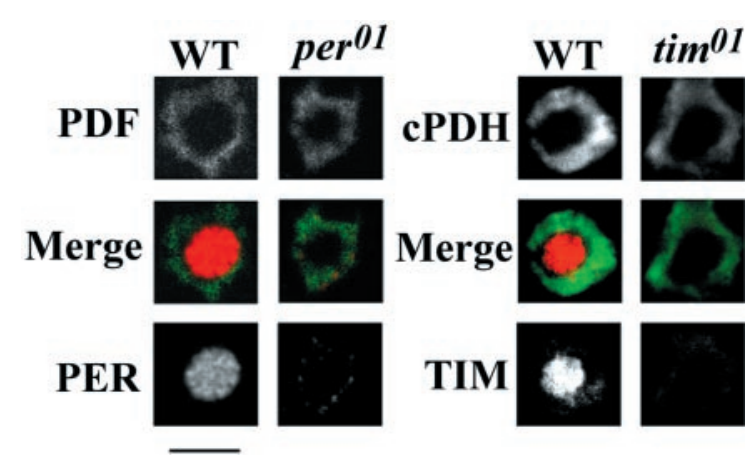

Figure 1. Expression of PER and TIM in the vLNs of wild-type (WT) and mutant flies. Typical vLNs of wild-type (Canton S), per ${ }^{01}$, and tim $^{01}$ null mutant flies dissected at ZT 00/24 are shown. Scale bar, $5 \mu \mathrm{m}$.

small or large vLNs in each sampled brain hemisphere, and the means and SEs of all brains at a particular ZT are reported in the graphs.

\section{RESULTS}

A subset of vLNs are the only neurons in the AMe to express the neuropeptide PDF and can be identified immunocytochemically through the use of antisera raised against PDF (Renn et al., 1999) or its crustacean ortholog $\mathrm{cPDH}$ (Helfrich-Forster, 1996). We used whole-mount immunocytochemistry and double-labeled brains for PDF and PER or for $\mathrm{CPDH}$ and TIM. Because the vLNs are the only cells in the AMe to express PDF, this doublelabel approach reveals only these $\sim 16$ neurons in this brain region. Moreover, the anti-neuropeptide signal is restricted to the cytoplasm, allowing us to delineate the nuclei of these neurons in optical sections of vLN cell bodies. Thus, the locations of the vLN nuclei were determined independently of their PER or TIM staining.

Figure 1 shows the results of such double labeling for wild-type (Canton S) and mutant per ${ }^{o 1}$ and tim $^{\text {o1 }}$ flies entrained to a $12 \mathrm{hr}$ LD cycle and dissected at ZT 00. As expected from previous studies (Vosshall et al., 1994; Curtin et al., 1995; Hunter-Ensor et al., 1996; Myers et al., 1996), wild-type flies showed exclusively nuclear PER and TIM staining at this time (Fig. 1). Also as expected, flies homozygous for loss of function $\mathrm{per}^{\mathrm{OI}}$ or $\mathrm{tim}^{\mathrm{OI}}$ mutations showed nearly undetectable PER or TIM signal, respectively (Fig. 1). This verifies that the antibodies are specific for these proteins.

\section{PER and TIM expression in the large vLNs throughout the day}

The large and small vLNs have different projection patterns in the central brain and are thought to subserve different functions (Helfrich-Forster and Homberg, 1993; Kaneko and Hall, 2000). For example, in flies homozygous for a mutation in a gene encoding the circadian photoreceptor CRY (cryptochrome), the small vLNs maintain robust cycling of per and tim mRNA, whereas the large neurons lack this molecular rhythm (Stanewsky et al., 1998). In the early stages of this study, we concentrated on the large vLNs.

In a daytime series of large vLNs, nuclear PER was visible throughout most of the day and did not disappear completely until after ZT 10 (Fig. $2 A$ ). Nuclear TIM disappeared by ZT 2 (Fig. 2B). Mean pixel intensities of both nuclear and cytoplasmic PER and TIM staining were quantified using NIH Image and plotted as a function of time (Fig. $2 C, D$ ). The pattern of TIM disappearance fit very well with published Western blot and 


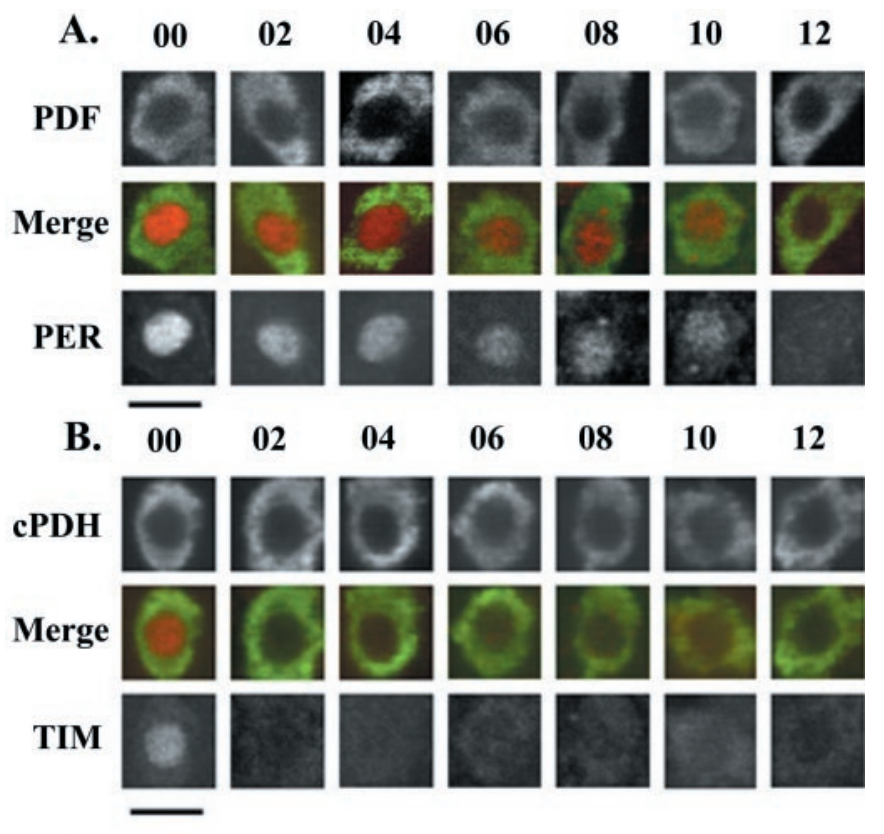

C.

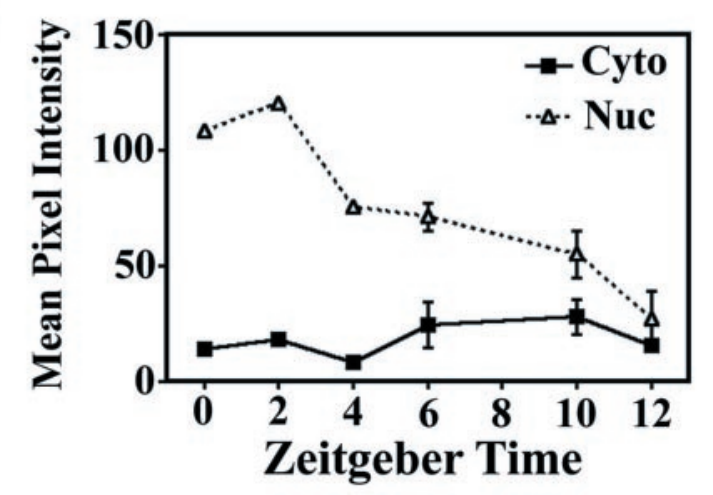

D.

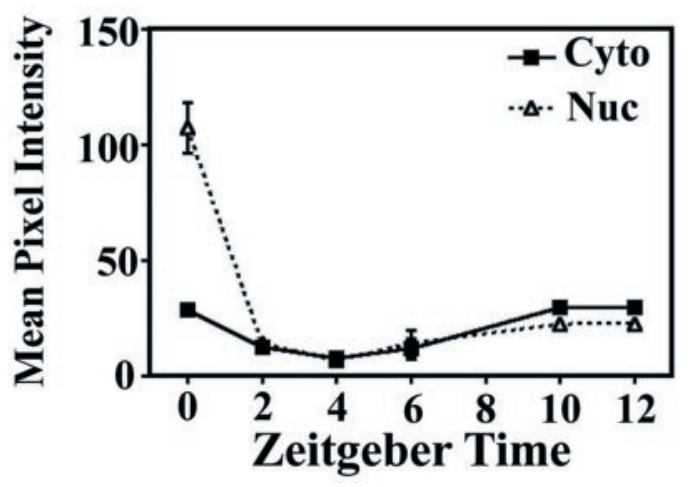

Figure 2. Daytime expression of PER and TIM in the large vLNs. $A$, Typical optical sections of large vLNs stained for PDF and PER. ZTs are indicated above each optical section. B, Typical optical sections of large vLNs stained for $\mathrm{CPDH}$ and TIM. Scale bars, $5 \mu \mathrm{m}$. $C$, Quantification of cytoplasmic (Cyto) and nuclear (Nuc) PER staining in the large vLNs as a function of ZT. D, Quantification of cytoplasmic and nuclear TIM staining in the large vLNs as a function of ZT. For each ZT, five brains were processed for each protein, and four large vLNs were imaged from one hemisphere of each brain.

histochemical data (Zerr et al., 1990; Zeng et al., 1996). PER immunoreactivity remained in the nuclei of the large vLNs slightly longer than predicted by whole-head Western blots. A full replicate series with the same number of flies gave the same pattern of nuclear PER and TIM disappearance (data not shown).

\section{Patterns of PER and TIM expression and nuclear accumulation differ throughout the night in the large vLNs}

A time course of night-time PER and TIM expression was determined for ZTs 12-24 in the manner described above. Surprisingly, the subcellular localization patterns of PER and TIM differed dramatically through most of the night. When PER was first detected at approximately ZT 16, it was already distributed uniformly in the cytoplasm and the nucleus. It then became predominantly nuclear by ZT 19 and remained exclusively nuclear for the rest of the night (Fig. 3A). Significant levels of TIM were also evident by ZT 16, but this protein remained cytoplasmic through ZT 18. It started to appear in the nucleus by ZT 19 but was not predominantly nuclear until ZT 21 (Fig. 3B). Both PER and TIM were found only in the nucleus at ZT 00 (Figs. 1, 3A,B).

Figure $3 C, D$ shows quantifications of nuclear and cytoplasmic PER and TIM intensities through the night. These two proteins increase in parallel starting at approximately ZT 16, but TIM remains in the cytoplasm for several hours until ZT 19 (Fig. 3D). The apparent nuclear TIM signal seen before ZT 19 in the quantification is likely caused by out-of-focus emission from cytoplasmic TIM above and below the optical section toward the edges of the nucleus (Fig. 3A,C,E). This conclusion is based on similar low-level signals seen at the edge of the nucleus in optical sections of PDF, cPDH, and TIM immunostaining (Fig. 3E). Antisera against PDF and cPDH detect a secreted protein that is presumably stored in vesicles. These antisera are therefore unlikely to bind nuclear peptides. Even if the nuclear signal reflects the presence of some nuclear TIM from ZT 16-18, it is clear that the majority of TIM is cytoplasmic, whereas the PER signal was equally strong in both compartments.

Although our method of quantification likely underestimates the differences between PER and TIM seen the images, we feel that it allows for objectivity in communicating the major trends in the data. Furthermore, we suggest that this method of quantification is preferable to the common practice of classifying stained cells into categories (e.g., cytoplasmic staining, cytoplasmic/nuclear staining, nuclear staining) and tallying the proportion of neurons in each category (Curtin et al., 1995).

A complete time course of a second set of brains gave the same results, including the enigmatic dip in nuclear PER at ZT 19 (data not shown). The cause of this drop in PER intensity is unknown.

To verify that PER accumulated in the nuclei of large vLNs before TIM, we labeled whole-mount brains dissected at ZT 17 using different PER and TIM antisera from those used in the series above. Again we found that PER was expressed uniformly throughout the cell, whereas TIM was restricted to the cytoplasm (data not shown). Because all four antibodies were polyclonal, it is unlikely that the apparent difference in PER and TIM nuclear accumulation is caused by epitope masking of nuclear TIM during the early evening. We also double-labeled brains that were dissected at ZT 17 for PER and TIM. Consistent with the above results, PER was expressed throughout the cytoplasm and nucleus of the large vLNs, whereas TIM was confined to the cytoplasm (Fig. $3 F$ ). We conclude that PER is not present in the nucleus as an obligate PER/TIM heterodimer before ZT 19. At earlier times, PER is present in the nucleus without stoichiometric amounts of TIM. However, these results do not preclude a cata- 
A.

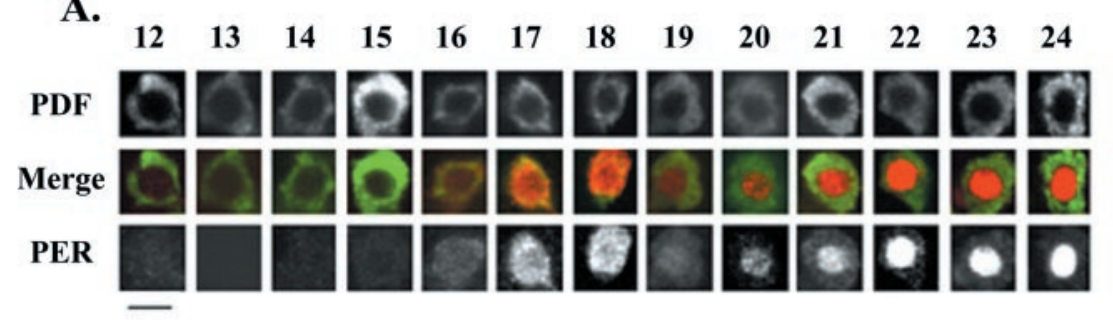

B.

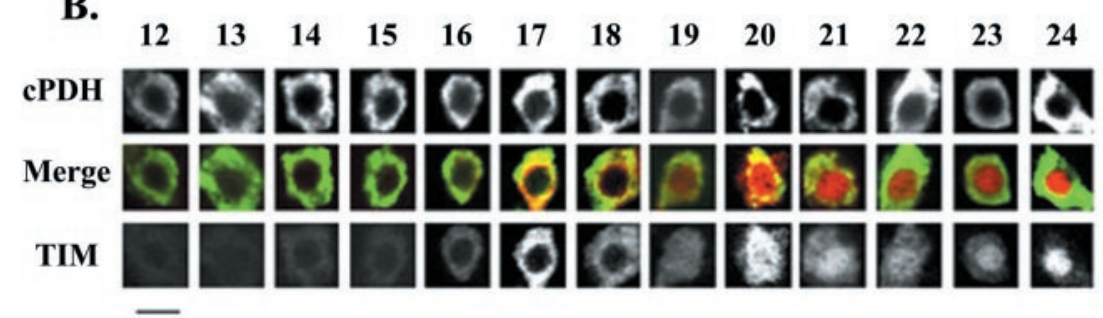

F.

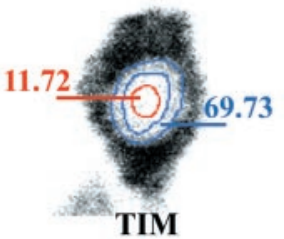

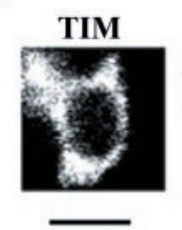

C.

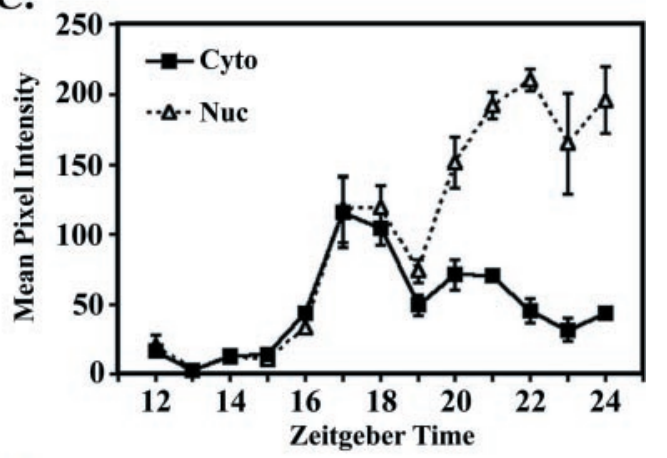

D.

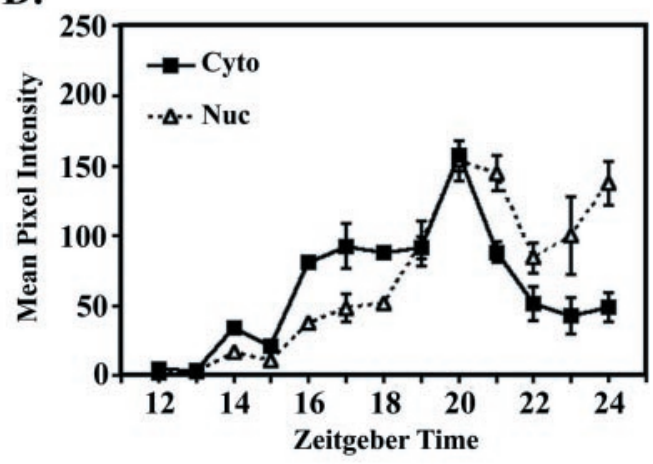

Figure 3. Night-time expression of PER and TIM in the large vLNs. $A$, Typical optical sections of large vLNs stained for PDF and PER. ZTs are indicated above each set of optical sections. $B$, Typical optical sections of large vLNs stained for cPDH and TIM. Scale bars, $5 \mu$ m. $C$, Quantification of cytoplasmic (Cyto) and nuclear (Nuc) PER staining in the large vLNs as a function of ZT. D, Quantification of cytoplasmic and nuclear TIM staining in the large vLNs as a function of ZT. For each ZT, five brains were processed for each protein and four large vLNs were imaged from one hemisphere of each brain. $E$, Inverted and quantified images of a large vLN double-labeled for cPDH and TIM at ZT 17. Staining values for the edge (blue) and center (red) of the nucleus are indicated. F, vLNs double-labeled for TIM and PER at ZT 17. Scale bar, $5 \mu \mathrm{m}$.

lytic role for TIM in PER nuclear entry (see Discussion) or the presence of small amounts of nuclear TIM before ZT 19.

\section{PER and TIM accumulate in the nuclei of small vLNs sequentially but in a manner different from the large vLNs}

Although both the large and small vLNs express PER, TIM, and PDF, the small cells differ from the large vLNs in their axonal projection patterns and are suspected to be the key pacemaker neurons of the CNS (Helfrich-Forster, 1998). The small vLNs, double-labeled for $\mathrm{CPDH} / \mathrm{PER}$ and $\mathrm{cPDH} / \mathrm{TIM}$ at key ZTs, are shown in Figure 4. PER was present throughout both cellular compartments in the small neurons at ZT 18, whereas TIM was largely restricted to the cytoplasm through ZT 20 (Fig. 4A,B), indicating that these proteins also accumulate sequentially in the nuclei of the small vLNs. However, two aspects of PER and TIM nuclear accumulation in the small vLNs differed from that seen in the large neurons. First, at ZT 16, PER was initially restricted to the cytoplasm (Fig. $4 A$ ), unlike the uniform distribution evident in the large vLNs (Fig. $3 A$, ZT 16). Second, the TIM signal was still predominantly cytoplasmic as late as ZT 20 in the small neurons (Fig. 4B, ZT 20), whereas the large neurons expressed TIM uniformly throughout both cell compartments by this time (Fig. $3 B$ ). The quantification of PER and TIM expression in the small neurons is shown in Figure $4 C, D$.

These results suggested that PER and TIM nuclear accumulation in the small vLNs phase-lags the large cells. To confirm this interpretation, we constructed a time course of PER and TIM expression in the large and small neurons from the same set of brains dissected every $2 \mathrm{hr}$ throughout the night (Fig. 5). As described above, PER was predominantly cytoplasmic in the small vLNs at ZT 16, whereas the large cells expressed this protein in both cellular compartments (Fig. 5A). The patterns of PER expression in the two cell types were essentially identical thereafter. Both groups of neurons expressed PER throughout the nucleus and cytoplasm at ZT 18 and displayed a predominantly nuclear PER signal at ZT 20 (Fig. 5A).

The timing of TIM nuclear accumulation differed markedly between the large and small vLNs. Although the large neurons displayed strong nuclear TIM at ZT 20, this protein was still cytoplasmic in the small cells at this time (Fig. 5B, ZT 20). TIM only became predominantly nuclear in the small cells at the very end of the night (ZT 24). Thus, the phase of TIM nuclear accumulation in the small neurons is delayed 3-4 hr relative to the large vLNs (Fig. 5B). The cell-specific differences in phase angles of PER and TIM nuclear accumulation also support the conclusion that these proteins are not accumulating in the nuclei of these neurons as obligate heterodimers.

\section{PER and TIM expression in the vLNs under constant conditions}

Behavioral rhythms and the molecular oscillations that underlie them persist in the absence of environmental time cues. We were interested in determining whether the vLNs showed similar patterns of PER and TIM accumulation under constant conditions. To this end, we assayed the distributions of these proteins in the large and small vLNs throughout a free-running circadian cycle. Flies were entrained to a $12 \mathrm{hr}$ LD cycle for 3-5 d and then kept 

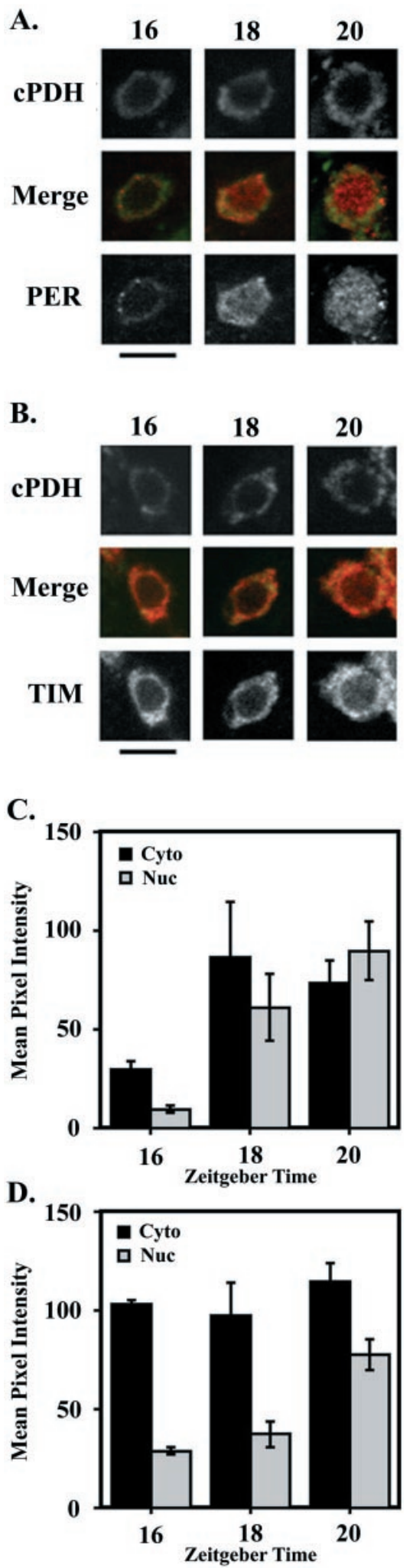

Figure 4. Night-time expression of PER and TIM in the small vLNs at ZTs 16,18 , and 20. $A$, Typical optical sections of small vLNs stained for cPDH and PER. ZTs are indicated above each set of optical sections. $B$, Typical optical sections of small vLNs stained for cPDH and TIM. Scale bars, $5 \mu \mathrm{m}$. $C$, Quantification of cytoplasmic (Cyto) and nuclear (Nuc) PER staining in the small vLNs. $D$, Quantification of cytoplasmic and nuclear TIM staining in the small vLNs. The quantification is based on images of four brains per time point per protein and three to four small vLNs per brain.
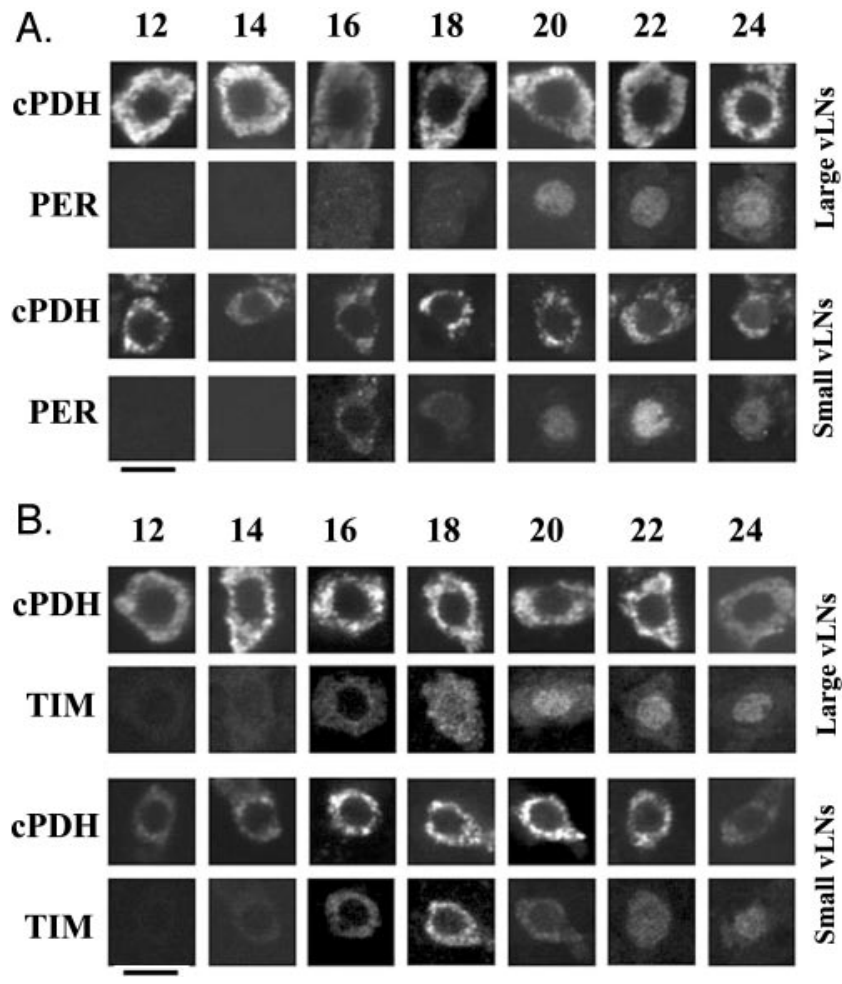

Figure 5. Comparison of nuclear accumulation in the large and small vLNs throughout the night. $A$, Typical optical sections of large and small vLNs stained for $\mathrm{CPDH}$ and PER. ZTs are indicated above each set of optical sections. $B$, Typical optical sections for large and small vLNs stained for cPDH and TIM. Scale bars, $5 \mu \mathrm{m}$.

under constant darkness (DD) and temperature $\left(25^{\circ} \mathrm{C}\right)$. Brains were dissected at various time points between circadian time (CT) 00 and 24 of the first circadian day (where CT 00/24 corresponds to the time of lights-on during the LD cycle of the preceding day).

Yang and Sehgal (2001) reported that the small vLNs but not the large cells express TIM rhythmically in DD. Our DD time course confirms and extends their observation by examining cells during the initial hours of DD exposure, by following PER as well as TIM, and by monitoring the subcellular distribution of both proteins (Figs. 6, 7). Indeed, TIM disappears from the nuclei of both the large and small cells by CT 02 in a manner similar to the early morning hours in LD (compare Fig. $6 A, B$ with $2 B$ ). In the large cells, TIM signal remained detectable but low for the rest of the circadian cycle (Fig. 6A,C). As expected, only the small neurons displayed a reaccumulation of TIM throughout the subjective night (Fig. 6B,D, CT 12-24). Cytoplasmic TIM first appeared at CT 16 and nuclear accumulation was first detected at CT 22 (Yang and Sehgal, 2001).

Our results showed a lack of PER cycling in the large vLNs (Fig. 7). In both the large and small cells, nuclear PER slowly wanes through most of the subjective day (Fig. 7, CT 00-12). In contrast to TIM, however, PER failed to disappear completely from the nuclei of large vLNs and was maintained at low, relatively constant levels throughout the subjective night (Fig. 7A). We suspected that the absence of PER signal at CT 20 was attributable to poorly stained preparations at this time point. Indeed, a replication of this CT revealed low levels of nuclear PER (data not shown). In the small vLNs, PER had disappeared from nuclei by the end of the subjective day (Fig. 7B, CT 10-12). 

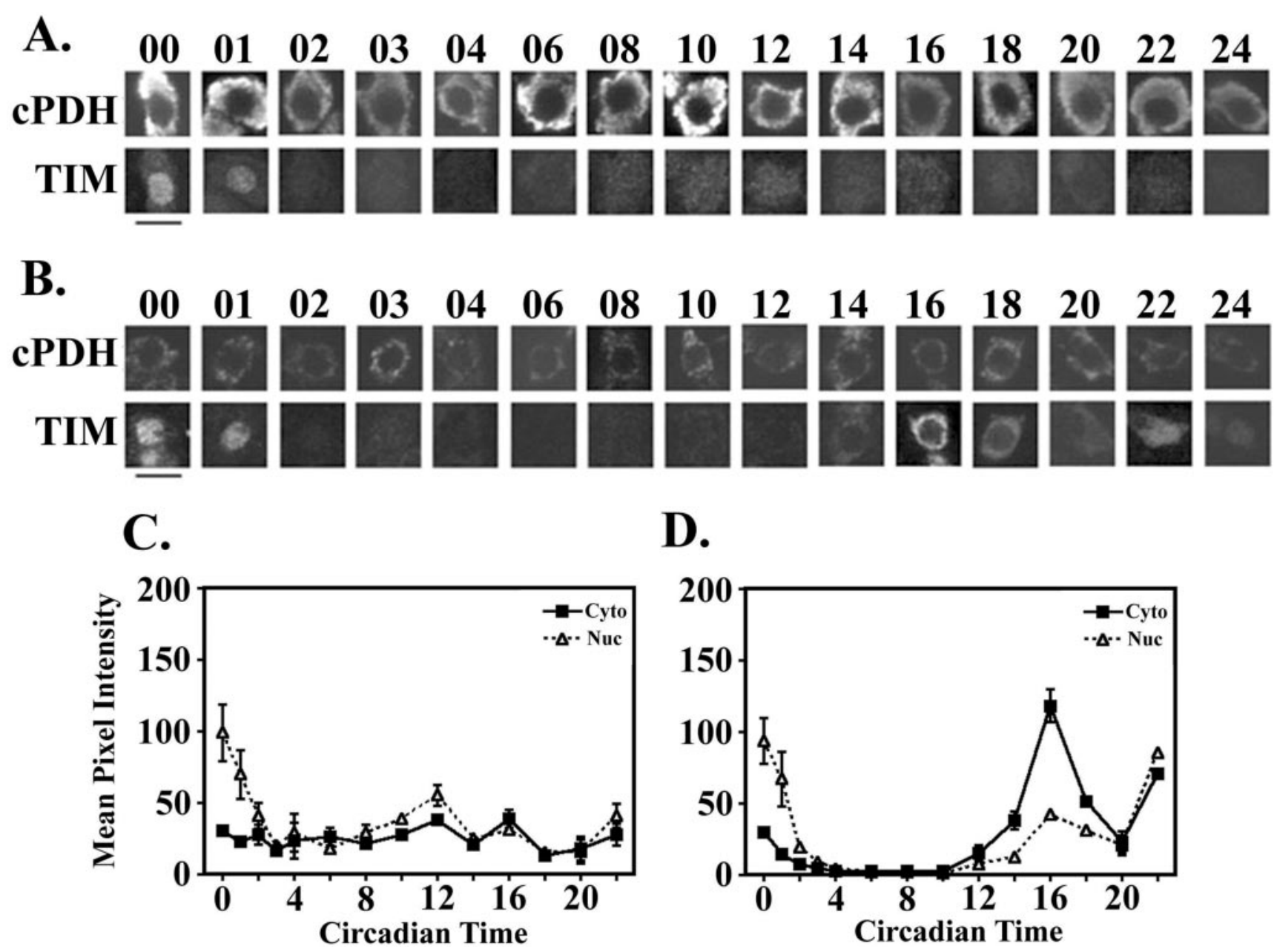

Figure 6. TIM expression in the large and small vLNs under constant darkness and temperature. $A$, Typical optical sections of large vLNs stained for cPDH and TIM. CTs are indicated above each set of optical sections. $B$, Typical optical sections of small vLNs stained for cPDH and TIM. Scale bars, $5 \mu \mathrm{m}$. C, Quantification of cytoplasmic (Cyto) and nuclear (Nuc) TIM staining in the large vLNs. D, Quantification of cytoplasmic and nuclear TIM staining in the small vLNs. The quantifications are based on images of four brains per time point per protein and three to four large or small vLNs per brain.

They began to reaccumulate PER at CT 16, with some nuclear signal evident at CT 18 and strong nuclear accumulation apparent by CT 22 (Fig. 7B,D).

\section{DISCUSSION}

We used antisera against the circadian clock proteins PER and TIM to analyze the temporal regulation of PER and TIM expression and the subcellular localization of these proteins in key pacemaker neurons of the adult brain. These cells, a subset of the vLNs of the AMe of Drosophila, play a central role in the control of behavioral rhythms (Helfrich-Forster, 1998; Renn et al., 1999). Unexpectedly, we found marked differences in the timing of PER and TIM nuclear localization. PER accumulated in the nucleus during the early night, whereas TIM was restricted to the cytoplasm of the vLNs through much of that time. Also, the two classes of PDF neurons, the large and small vLNs, had a similar timing of PER nuclear accumulation but differed by $\sim 3 \mathrm{hr}$ in TIM nuclear accumulation. These features are not predicted by the current molecular model of circadian clock function in Drosophila and are inconsistent with the hypothesis that PER and TIM function as obligate heterodimers throughout the night.

One concern of an immunocytochemical study is that differ- ences in staining intensity for two epitopes may reflect a disparity in antibody affinity rather than differences in epitope abundance. We have attempted to allay this concern by using two to three different PER and TIM antibodies. Similar intensities and patterns of staining are seen for all of the antibodies used in this study, suggesting that differential antibody affinity is not responsible for the differences in staining observed for these two proteins. The best estimate for the relative abundance of PER and TIM comes from the analysis of whole-head extracts by Zeng et al. (1996), who showed that the TIM/PER ratio is $\sim 2: 1$ at ZT 16 and $1: 1$ by $\mathrm{ZT} 23$. A variation of this magnitude in the vLNs would be consistent with the relative intensities of staining observed in this study (Figs. 3C,D, 4C,D). Given this transient disparity between TIM and PER abundance, the persistence of strong cytoplasmic TIM signals at times when PER is predominantly nuclear must not be considered as evidence that these proteins enter the nucleus sequentially. Rather, it is the differing profiles of TIM and PER immunoreactivity in the nuclei of the vLNs on which we base this conclusion.

Several lines of evidence had suggested that PER and TIM are transported into nuclei as a dimeric complex. Biochemical assays 

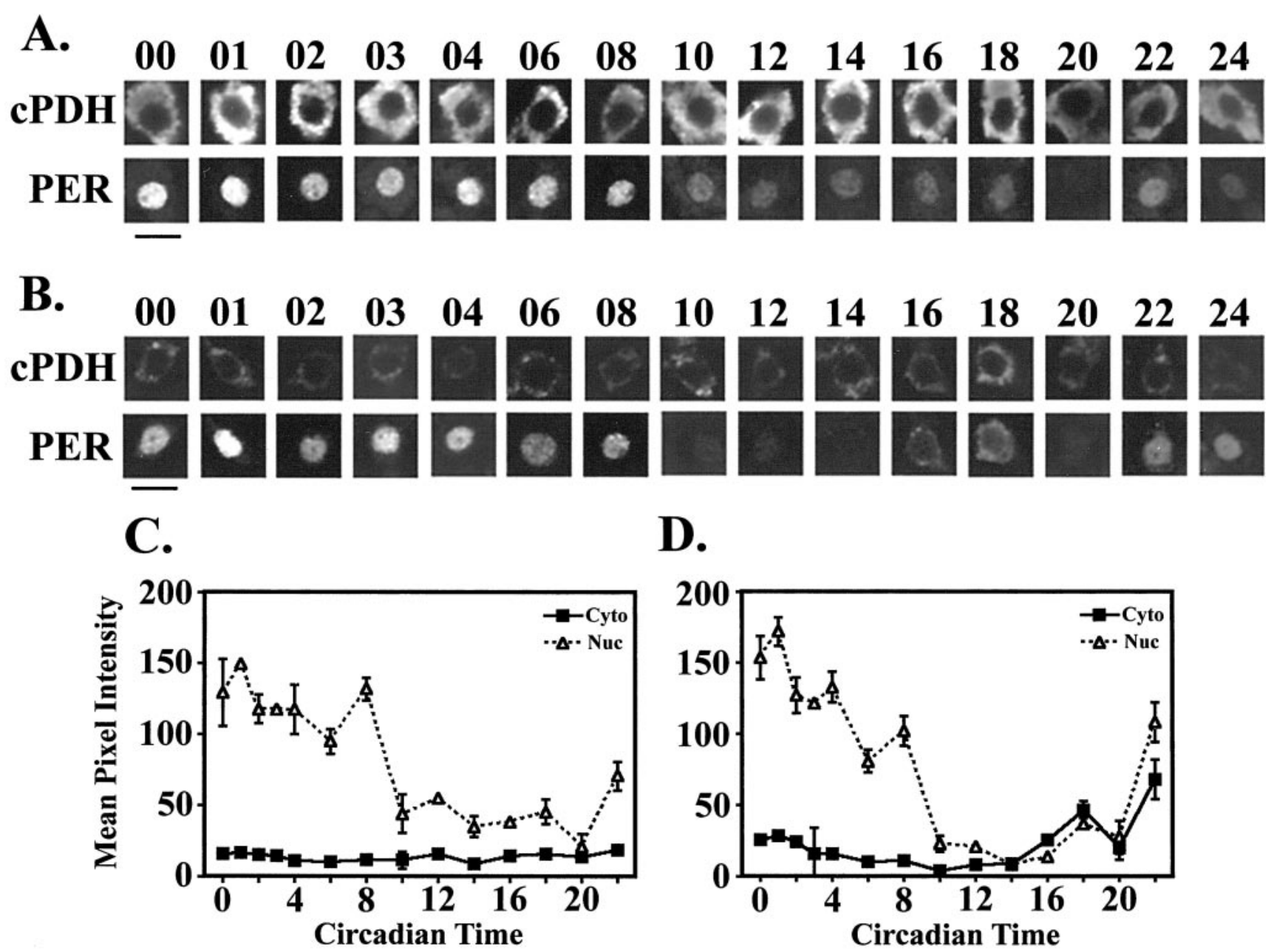

Figure 7. PER expression in the large and small vLNs under constant darkness and temperature. $A$, Typical optical sections of large vLNs stained for cPDH and PER. CTs are indicated above each set of optical sections. B, Typical optical sections of small vLNs stained for cPDH and PER. Scale bars, $5 \mu \mathrm{m}$. $C$, Quantification of cytoplasmic (Cyto) and nuclear (Nuc) PER staining in the large vLNs. D, Quantification of cytoplasmic and nuclear PER signal in the small vLNs. The quantifications are based on images of four brains per time point per protein and three to four large or small vLNs per brain.

in fly-head extracts show that PER and TIM are present as heterodimers throughout much of the night (Lee et al., 1996; Zeng et al., 1996), but the data did not exclude the fact that a fraction of PER and TIM are monomeric or complexed with other proteins. Importantly, it is also uncertain what fraction of the PER/TIM heterodimers are present in vivo and what fraction form in vitro during extract preparation and analysis. Furthermore, these extracts reflect thousands of cells of many different types, including all of the photoreceptors from the compound eye. These contribute the majority of the PER and TIM to the homogenate, effectively swamping out the contribution of the vLNs. There is therefore no reason to expect that the biochemistry of the vLNs will be identical to what has been observed in whole-head extracts. In flies, Vosshall et al. (1994) observed little or no nuclear PER without TIM, but this observation is likely attributable to PER instability and consequent low PER levels in mutants that lack TIM (Price et al., 1995). Finally, Saez and Young (1996) established that the coexpression of PER and TIM is required for the nuclear transport of both proteins in cultured S2 cells. Although these cells have not been shown to express PER and TIM rhythmically, this codependence fits well with the notion that PER and TIM enter the nucleus as a heterodimeric complex. Based on these considerations, we expected that our time course would reveal the simultaneous nuclear accumulation of PER and TIM in the vLNs. Instead, our data showed that PER appears in nuclei at least $3 \mathrm{hr}$ before TIM, indicating that most, and perhaps all, nuclear PER is not complexed with TIM at these times.

In the large vLNs, PER is already nuclear as well as cytoplasmic when it is first detectable at ZT 16. This result is somewhat different from a previous report, which could not detect nuclear PER in presumptive vLNs until ZT 18 (Curtin et al., 1995). In our study, PER first shows clear nuclear localization within the small vLNs at ZT 18; before that time, PER is cytoplasmic. This pattern of PER expression, although less intense in the present report, is reminiscent of the description of PER expression by Curtin et al. (1995). Thus, this previous study (which did not distinguish between the large and small vLNs) may have been observing only the small cells. Furthermore, the weaker signal observed here is most likely caused by the use of fluorescently labeled secondary antisera in place of the enzyme-conjugated antisera used by Curtin et al. (1995).

In the large vLNs, we first detected nuclear PER at times that correlate well with the onset of repression in head extracts. This begins at approximately ZT $15-17$ by biochemical criteria (So and Rosbash, 1997). The discrepancy between the timing of PER 
nuclear accumulation in the small cells and the onset of transcriptional repression in whole-head extracts might once again reflect differences between the cells and tissues that contribute to the biochemical data (see above).

In contrast to PER, TIM is not nuclear until after ZT 18. This difference with PER is especially prominent in the small cells, where TIM remains cytoplasmic until ZT 22. In both cell types, TIM is degraded in the early morning and is therefore nuclear for only a short time. In other words, TIM becomes nuclear well after transcriptional repression is thought to begin and is degraded well before the next cycle of transcription begins anew.

A central feature of the molecular model of the Drosophila clock is that PER and TIM function as obligate heterodimers in their nuclear transport as well as in the repression of Clock/ Cycle-based transcription (Williams and Sehgal, 2001). However, the different times of nuclear accumulation suggest that PER and TIM are transported independently to nuclei. The lack of highresolution data for the doubletime kinase (Kloss et al., 2001) makes it difficult to assess whether the doubletime nuclear accumulation pattern correlates better with that of PER or of TIM. Importantly, our results do not preclude a role for TIM in the nuclear entry of PER. For example TIM could act catalytically in the cytoplasm to potentiate some required PER phosphorylation event. Moreover, PER and TIM could still enter nuclei as PER/ TIM heterodimers, but this would require the subsequent export of TIM to the cytoplasm to explain the apparent absence of nuclear TIM during the middle of the night.

In biochemical assays, PER and TIM are both capable of blocking per and tim transcription in the absence of the other, suggesting that repression does not require the PER/TIM heterodimer (Lee et al., 1999; Schotland et al., 2000). Our data indicate that little or no TIM is present in the nuclei of vLNs during times associated with repression in whole-head extracts, suggesting that PER might influence transcription independently of TIM. Work by Rothenfluh et al. (2000) suggests that PER is still capable of repressing transcription after a mutant form of TIM, TIM ${ }^{\mathrm{UL}}$, is degraded by light exposure. The authors conclude that PER can repress transcription in the absence of TIM and suggest that TIM-independent repression by PER normally occurs after dawn. However, they had no reason to challenge the notion that the PER/TIM heterodimer is the agent of nuclear entry and the initiator of repression (Rothenfluh et al., 2000). Our data indicate that the TIM-independent repression by PER also applies to the onset of repression in the middle of the night. TIM may therefore play no direct role in the feedback repression of PER and TIM transcription.

A previous study described the persistence of TIM oscillations in the small vLNs but the absence of oscillations in the large vLNs under DD conditions (Yang and Sehgal, 2001). We have confirmed this observation, but our data extend this result in several important ways. We have assayed PER as well as TIM and have followed these two proteins throughout the first DD cycle. This provides a finer temporal resolution and leads to a surprising conclusion: that the molecular oscillation in the large vLNs appears to arrest during the first subjective day. Based on the patterns of PER and TIM, these cells stop in a state that corresponds to ZT 8-10 under LD conditions.

The individual features of PER and TIM distribution in the large and small vLNs can be added to a growing list of differences between these two classes of PDF-expressing neurons (see discussion in Park et al., 2000). These cell-specific differences in PER and TIM nuclear accumulation underscore the importance of studying the brain clock in situ and may presage an even more profound heterogeneity among other clock-containing neurons within the brain. This view also reflects the fact that the PDFpositive vLNs represent a minority of clock gene-expressing cells in the central brain (Helfrich-Forster, 1996; Kaneko and Hall, 2000). One might therefore expect that the circadian system will vary to an even greater degree among other clock neurons. Such diversity might offer clues as to how different groups of clock neurons interact to create the temporal complexity of behavioral rhythms.

\section{REFERENCES}

Allada R, White NE, So WV, Hall JC, Rosbash M (1998) A mutant Drosophila homolog of mammalian Clock disrupts circadian rhythms and transcription of period and timeless. Cell 93:791-804.

Curtin KD, Huang ZJ, Rosbash M (1995) Temporally regulated nuclear entry of the Drosophila Period protein contributes to the circadian clock. Neuron 14:365-372.

Darlington TK, Wager-Smith K, Ceriani MF, Staknis D, Gekakis N, Steeves TDL, Weitz CJ, Takahashi JS, Kay SA (1998) Closing the circadian loop: CLOCK-induced transcription of its own inhibitors per and tim. Science 280:1599-1603.

Dircksen H, Zahnow CA, Gaus G, Keller R, Rao KR, Riehm JP (1987) The ultrastructure of nerve endings containing the pigment-dispersing hormone (PDH) in crustacean sinus glands: identification by an antiserum against synthetic PDH. Cell Tissue Res 250:377-387.

Dunlap JC (1999) Molecular bases for circadian clocks. Cell 96:271-290.

Ewer J, Frisch B, Hamblen-Coyle MJ, Rosbash M, Hall JC (1992) Expression of the period clock gene within different cell types in the brain of Drosophila adults and mosaic analysis of these cells' influence on circadian behavioral rhythms. J Neurosci 12:3321-3349.

Frisch B, Hardin PE, Hamblen-Coyle MJ, Rosbash M, Hall JC (1994) A promoterless period gene mediates behavioral rhythmicity and cyclical per expression in a restricted subset of the Drosophila nervous system. Neuron 12:555-570.

Hardin PE, Hall JC, Rosbash M (1990) Feedback of the Drosophila period gene product on circadian cycling of its messenger RNA levels. Nature 343:536-540

Hardin PE, Hall JC, Rosbash M (1992) Behavioral and molecular analyses suggest that circadian output is disrupted by disconnected mutants in D. melanogaster. EMBO J 11:1-6.

Helfrich-Forster C (1995) The period clock gene is expressed in central nervous system neurons which also produce a neuropeptide that reveals the projections of circadian pacemaker cells within the brain of Drosophila melanogaster. Proc Natl Acad Sci USA 92:612-616.

Helfrich-Forster C (1996) Drosophila rhythms: from brain to behavior. Semin Cell Dev Biol 7:791-802.

Helfrich-Forster C (1998) Robust circadian rhythmicity of Drosophila melanogaster requires the presence of lateral neurons: a brain-behavioral study of disconnected mutants. J Comp Physiol [A] 182:435-453.

Helfrich-Forster C, Homberg U (1993) Pigment-dispersing hormoneimmunoreactive neurons in the nervous system of wild-type Drosophila melanogaster and of several mutants with altered circadian rhythmicity. J Comp Neurol 337:177-190.

Hunter-Ensor M, Ousley A, Sehgal A (1996) Regulation of the Drosophila protein timeless suggests a mechanism for resetting the circadian clock by light. Cell 84:677-685.

Kaneko M, Hall JC (2000) Neuroanatomy of cells expressing clock genes in Drosophila: transgenic manipulation of the period and timeless genes to mark the perikarya of circadian pacemaker neurons and their projections. J Comp Neurol 422:66-94.

Kloss B, Rothenfluh A, Young MW, Saez L (2001) Phosphorylation of PERIOD is influenced by cycling physical associations of DOUBLETIME, PERIOD, and TIMELESS in the Drosophila clock. Neuron 30:699-706.

Konopka RJ, Benzer S (1971) Clock mutants of Drosophila melanogaster. Proc Natl Acad Sci USA 68:2112-2116.

Lee C, Parikh V, Itsukaichi T, Bae K, Edery I (1996) Resetting the Drosophila clock by photic regulation of PER and a PER-TIM complex. Science 271:1740-1744.

Lee C, Bae K, Edery I (1999) PER and TIM inhibit the DNA binding activity of a Drosophila CLOCK-CYC/dBMAL1 heterodimer without disrupting formation of the heterodimer: a basis for circadian transcription. Mol Cell Biol 19:5316-5325.

Lee C, Etchegaray J, Cagampang FRA, Loudon ASI, Reppert SM (2001) Posttranslational mechanisms regulate the mammalian circadian clock. Cell 107:855-867.

Myers MP, Wager-Smith K, Rothenfluh-Hilfiker A, Young MW (1996) Light-induced degradation of TIMELESS and entrainment of the Drosophila circadian clock. Science 271:1736-1740. 
Park JH, Helfrich-Forster C, Lee G, Liu L, Rosbash M, Hall JC (2000) Differential regulation of circadian pacemaker output by separate clock genes in Drosophila. Proc Natl Acad Sci USA 97:3608-3613.

Price JL, Dembinska ME, Young MW, Rosbash M (1995) Suppression of PERIOD protein abundance and circadian cycling by the Drosophila clock mutation timeless. EMBO J 14:4044-4049.

Renn SC, Park JH, Rosbash M, Hall JC, Taghert PH (1999) A pdf neuropeptide gene mutation and ablation of PDF neurons each cause severe abnormalities of behavioral circadian rhythms in Drosophila. Cell 99:791-802.

Rothenfluh A, Young MW, Saez L (2000) A TIMELESS-independent function for PERIOD proteins in the Drosophila clock. Neuron 26:505-514.

Rutila JE, Suri V, Le M, So WV, Rosbash M, Hall JC (1998) CYCLE is a second bHLH-PAS clock protein essential for circadian rhythmicity and transcription of Drosophila period and timeless. Cell 93:805-814.

Saez L, Young MW (1996) Regulation of nuclear entry of the Drosophila clock proteins period and timeless. Neuron 17:911-920.

Schotland P, Hunter-Ensor M, Lawrence T, Sehgal A (2000) Altered entrainment and feedback loop function effected by a mutant period protein. J Neurosci 20:958-968

Sehgal A, Price JL, Man B, Young MW (1994) Loss of circadian behavioral rhythms and per RNA oscillations in the Drosophila mutant timeless. Science 263:1603-1606.
Sehgal A, Rothenfluh-Hilfiker A, Hunter-Ensor M, Chen Y, Myers MP, Young MW (1995) Rhythmic expression of timeless: a basis for promoting circadian cycles in period gene autoregulation. Science 270:808-810.

So WV, Rosbash M (1997) Post-transcriptional regulation contributes to Drosophila clock gene mRNA cycling. EMBO J 16:7146-7155.

Stanewsky R, Kaneko M, Emery P, Beretta B, Wager-Smith K, Kay SA, Rosbash M. Hall JC (1998) The $c r y^{b}$ mutation identifies cryptochrome as a circadian photoreceptor in Drosophila. Cell 95:681-692.

Suri V, Lanjuin A, Rosbash M (1999) TIMELESS-dependent positive and negative autoregulation in the Drosophila circadian clock. EMBO J 18:675-686.

Vosshall LB, Price JL, Sehgal A, Saez L, Young MW (1994) Block in nuclear localization of period protein by a second clock mutation, timeless. Science 263:1606-1609.

Williams JA, Sehgal A (2001) Molecular components of the circadian system in Drosophila. Annu Rev Physiol 63:729-755.

Yang Z, Sehgal A (2001) Role of molecular oscillations in generating behavioral rhythms in Drosophila. Neuron 29:453-467.

Zeng H, Qian Z, Myers MP, Rosbash M (1996) A light-entrainment mechanism for the Drosophila circadian clock. Nature 380:129-135.

Zerr DM, Hall JC, Rosbash M, Siwicki KK (1990) Circadian fluctuations of period protein immunoreactivity in the CNS and the visual system of Drosophila. J Neurosci 10:2749-2762. 